\title{
Redistribution of aquaporins 1 and 5 in the rat uterus is dependent on progesterone: a study with light and electron microscopy
}

\author{
Laura A Lindsay and Christopher R Murphy \\ School of Medical Sciences (Anatomy and Histology), The University of Sydney, Sydney, NSW 2006, Australia \\ Correspondence should be addressed to LA Lindsay; Email: laural@anatomy.usyd.edu.au
}

\begin{abstract}
During early pregnancy in the rat there is a dramatic reduction in luminal fluid which is associated with uterine receptivity for blastocyst implantation. This study investigates the presence and distributional changes of several members of the aquaporin (AQP) family in the rat uterus in response to hormonal regime. An increase in apical AQP5 protein expression was found in response to progesterone alone or in combination with oestrogen, which is similar to that seen at the time of implantation. AQP1 was found in endothelial cells of the endometrium and in the inner circular layer of smooth muscle, with maximal protein expression seen after three doses of progesterone plus $8 \mathrm{hr}$ of oestrogen treatment. These results, for the first time, show that the up-regulation of AQP5 in the apical plasma membrane of uterine epithelial cells and AQP1 in the inner circular layer of myometrium, is dependent on progesterone. Furthermore, unlike during normal pregnancy, there is no differential gradient of AQP5 expression between mesometrial and antimesometrial poles of the progesterone treated uterus. Hence it is suggested that the differential gradient of AQP5 is dependent on the presence of a blastocyst, in addition to the appropriate hormonal environment.

Reproduction (2006) 131 369-378
\end{abstract}

\section{Introduction}

Water transport across an epithelial barrier can occur by transcellular or paracellular means. The paracellular pathway of fluid transport between cells is mainly regulated by the tight junction complex (Claude \& Goodenough 1973). Transcellular fluid transport across cells can occur by diffusion alone or through specialised transporters, known as aquaporins (AQP). AQP are present in a variety of epithelia and exhibit a greater capacity for water movement when compared with simple diffusion alone (Agre et al. 2002). AQP1, 4 and 5 are water selective and therefore are members of the classical AQP family (Agre et al. 1998).

AQP1, a $28 \mathrm{kDa}$ protein initially isolated from red blood cells (Agre et al. 1987, Denker et al. 1988), has been localised to endothelial cells (Nielsen et al. 1993) as well as the inner circular layer of uterine smooth muscle (Lindsay \& Murphy 2004a). Furthermore, it was found that AQP1 was most concentrated within the mesometrial myometrium at the time of implantation in the rat (Lindsay \& Murphy 2004a). AQP4 is widely distributed in various tissue types, such as the kidney (Yamamoto \& Sasaki 1998, van Hoek et al. 2000), respiratory tract (Song et al. 2001) and brain (Nielsen et al. 1997b) but was absent from the pregnant rat uterus (Lindsay \& Murphy 2004b). AQP5 has mainly been localised in apical plasma membranes of various secretory glands including salivary glands and submucosal glands of the respiratory tract as well as type I pneumocytes (Nielsen et al. 1997a). AQP5 was found to be present on the apical plasma membrane of uterine epithelial cells at the time of implantation in the rat, with the greatest concentration of channels found in mesometrial uterine epithelial cells (Lindsay \& Murphy 2004b).

At the time of implantation in the rat, as a component of the complex alterations in uterine epithelial cells (Julian et al. 2005), there is a dramatic reduction in the volume of uterine luminal fluid, with the uterine lumen closing down resulting in close apposition between the uterine epithelium and trophoblastic cells of the implanting blastocyst (Enders \& Schlafke 1967). Freeze fracture studies demonstrated an increase in depth of the tight junctions and more branching of the tight junction strands at this time (Murphy 2000). Recent evidence has shown that AQP5 is redistributed to the apical plasma membrane of uterine luminal epithelial cells on days 6 and 7 of pregnancy (Lindsay \& Murphy 2004b). Hence it is likely that at the time of implantation in the rat, uterine fluid is transported from the lumen into the endometrial stroma by 
AQP channels present within the apical plasma membrane of luminal epithelial cells.

These uterine changes of early pregnancy are driven by ovarian steroid hormones. Oestradiol causes stromal oedema, beginning at the antimesometrial pole of the uterus while after several days of oestrogen, the uterine lumen is distended and full of fluid (Ljungkvist 1971a). Progesterone alone, or in combination with oestrogen results in closing down of the uterine lumen (Ljungkvist 1971b). Studies investigating tight junctions in luminal epithelial cells of the rat found that oestrogen led to 'leaky' tight junctions between these cells, while progesterone led to the formation of 'tight' tight junctions (Murphy et al. 1981). There is however no accepted explanation of this well established phenomenon of stromal oedema nor the regulation of uterine fluid volume by ovarian hormones.

Previous studies using immature rats indicate that AQP1 mRNA is up-regulated in response to administration of oestradiol (Li et al. 1997). Furthermore, the expression of AQP1 was found in the inner circular layer of myometrium in control and progesterone treated animals (Richard et al. 2003). Another study found AQP1 protein within both layers of the myometrium in all treatment groups with a slight increase in oestradiol treated mice and a slight decrease in the progesterone treated group, when compared with the control group (Jablonski et al. 2003). Animals treated with oestradiol or progesterone followed by oestradiol showed a biphasic response with expression initially in the inner circular layer of myometrium and 24 hrs later, a shift of AQP1 mRNA expression to the uterine stromal endothelial cells (Richard et al. 2003).

AQP2 was found to be absent from the pregnant mouse uterus (Richard et al. 2003). However, AQP2 was shown to be strongly regulated by oestrogen, with protein present in luminal and glandular epithelium as well as in the myometrium (Jablonski et al. 2003). AQP4 mRNA was not found in ovariectomised mice treated with either oil, oestradiol, progesterone or a combination of progesterone followed by oestradiol (Jablonski et al. 2003, Richard et al. 2003). In uterine luminal epithelial cells AQP5 mRNA was up-regulated after exposure of ovariectomised mice to progesterone followed by a nidatory dose of oestradiol (Richard et al. 2003), but was absent in the control or oestrogen treated mouse uterus (Jablonski et al. 2003).

It is thus evident that AQP channels are likely to be important in the implantation process of rodents during normal pregnancy but to date the hormonal control of this phenomenon is unknown. Previous studies have highlighted differences in AQP expression, particularly AQP5, between rats and mice at the time of implantation (Lindsay \& Murphy 2004b). Hence the aim of this current study is to investigate the hormonal regulation of AQP1, AQP4 and AQP5 protein expression in the ovariectomised and hormone treated rat model using light and electron microscopy techniques.

\section{Materials and Methods}

\section{Animals}

Twenty-five adult, female, virgin Wistar rats were used in this study and housed at $21^{\circ} \mathrm{C}$ with a $12 \mathrm{~h}$ light:darkness cycle and given food and water ad libitum. Animals 12-14 weeks old were bilaterally ovariectomised using a ventral approach under isoflurane anaesthesia and allowed to recover for at least 4 weeks. Rats were randomly divided into five groups each with five animals and treated with different hormonal regimes. All hormonal doses were within the normal physiological range (Ljungkvist 1971a, b). Hormones were dissolved in benzyl alcohol (Sigma, St Louis, MO, USA) and diluted in peanut oil to achieve levels as described below. Subcutaneous injections were given in the scruff of the neck. Animals in the control group were injected with $0.1 \mathrm{ml}$ peanut oil alone for three consecutive days. Animals in group 2 were injected with $0.5 \mu \mathrm{g} 17$ - $\beta$-oestradiol (Sigma) in $0.1 \mathrm{ml}$ peanut oil for three consecutive days. Group 3 animals were injected for three consecutive days with $5 \mathrm{mg}$ progesterone (Sigma) in $0.2 \mathrm{ml}$ peanut oil. All animals in these three groups were killed $24 \mathrm{~h}$ after the last injection with an intraperitoneal injection of sodium pentobarbitone (Nembutal, Merial Australia, Parramatta, NSW, Australia). Animals in the remaining groups were injected with $5 \mathrm{mg}$ progesterone for 2 days and $0.5 \mu \mathrm{g} 17-\beta$-oestradiol as well as $5 \mathrm{mg}$ progesterone on day 3 on opposite sides of the neck. Half of these animals were killed $8 \mathrm{~h}$ after the last injection with the remainder killed $24 \mathrm{~h}$ after the last injection. Uterine horns were excised from the euthanasised animals and randomly assigned for either light (LM) or electron microscope (EM) studies.

\section{Light microscopy}

Uterine tissue was immediately placed in ice-cold $0.1 \mathrm{M}$ phosphate buffer ( $\mathrm{pH} 7.4)$, cut into $5 \mathrm{~mm}$ blocks and immersed in OCT compound (Tissue Tek, Tarrance, CA, USA). Blocks were then immersed in supercooled isopentane (BDH Laboratory Supplies, Poole, England) before being stored in liquid nitrogen. Two blocks per animal were cut using a CM3050 cryostat (Leica, Heerbrugg, Switzerland) and $8 \mu \mathrm{m}$ thick sections were placed onto gelatin-coated slides. After briefly air drying, sections were fixed in acetone at $-20^{\circ} \mathrm{C}$ for at least $1 \mathrm{~h}$. Remaining steps were carried out at room temperature. After briefly air drying, sections were incubated in a pre-blocking solution of PBS containing 1\% BSA (Sigma). This solution was also used as diluent for all primary and secondary antibodies. Sections were then incubated in the primary antibody solution for at least $3 \mathrm{~h}$.

Primary antibodies used in this study were directed against unique sequences of rat AQP1, AQP4 or AQP5 (Alpha Diagnostics International, San Antonio, TX, USA) and were used at concentrations of $2.5,20$ and $3.3 \mu \mathrm{g} / \mathrm{ml}$ respectively. These members of the AQP family were 
chosen as they have previously been shown to be present in the pregnant and hormonally treated mouse uterus (Richard et al. 2003). Sections were washed in PBS and incubated for $1 \mathrm{~h}$ in FITC conjugated goat-anti-rabbit secondary antibodies at a concentration of $7.5 \mu \mathrm{g} / \mathrm{ml}$. From this point onwards sections were kept in the dark to prevent quenching of the fluorescent signal. Sections were then washed in PBS, mounted with Vectashield (Vector, Burlingame, CA, USA), cover slipped and visualised within $1 \mathrm{~h}$ of completing this protocol with a Diaplan microscope (Leica). Digital micrographs were taken using a Leica DC200 camera and micrographs were produced using Photoshop software (Adobe Systems, San Jose, CA, USA). Final magnifications were calculated using a stage micrometer.

\section{Electron microscopy}

The uterine horns randomly selected for electron microscopy (EM) processing were immediately fixed in freshly prepared $4 \%$ formaldehyde solution for $40 \mathrm{mins}$ during which time $5 \mathrm{~mm}$ and finally $1 \mathrm{~mm}$ rings were cut. Tissue was then washed in 0.1 M phosphate buffer $(\mathrm{pH} 7.4$ ) and from this point onwards all incubation steps were carried out at $4{ }^{\circ} \mathrm{C}$. Tissue was then dehydrated in graded alcohol, infiltrated with LR white resin (Sigma) containing $0.5 \%$ benzoin ethyl ether (Fluka, Buchs, Switzerland) embedded in gelatin capsules with fresh resin and finally polymerised with u.v. light.

An ultracut UCT ultramicrotome (Leica) was used to cut two blocks per animal. Silver-gold ultrathin sections were collected onto nickel grids and air dried. Prior to immunolabeling, sections were rehydrated and non specific staining was blocked by incubation in PBS containing $0.05 \mathrm{M}$ glycine followed by PBS containing $1 \%$ BSA. Sections were incubated overnight at $4{ }^{\circ} \mathrm{C}$ in solutions containing anti-AQP1 or anti-AQP5 antibodies. After washing in PBS containing 1\% BSA, sections were incubated for $2 \mathrm{~h}$ at room temperature in a solution of ultrasmall gold conjugated goat-anti-rabbit secondary antibodies (Aurion, Wageningen, Netherlands) at a concentration of $0.6-0.8 \mu \mathrm{g} / \mathrm{ml}$. Further washing was followed by post fixation in $2 \%$ glutaraldehyde in PBS, washed again and silver enhanced (Aurion) for 30-35 mins. Sections were washed in distilled water, air dried and viewed unstained using a JEM-1010 electron microscope operating at $80 \mathrm{kV}$ (Jeol, Tokyo, Japan)

\section{Controls}

Negative controls, in which the primary antibody was omitted, were run in parallel with all experimental runs. Four randomly selected sections for each animal in light microscopy (LM) studies and at least 2 randomly selected grids per animal in EM studies were processed as negative controls to detect any non specific binding of the secondary antibody.
Skeletal muscle sections were run in parallel with antiAQP4 immunofluorescence studies as a positive control.

Non-immune controls were carried out in LM and EM experiments by replacement of primary antibodies with normal purified rabbit IgGs (Sigma). In addition, randomly selected grids were processed without incubation with primary or secondary antibodies in order to detect any nonspecific silver enhancement.

\section{Results}

\section{Light microscopy}

AQP1 immunofluorescent staining is seen in endometrial blood vessels in all treatment groups, with a variation in AQP1 staining of the myometrium between treatment groups. An increase in AQP1 is seen in the inner circular layer of myometrium in ovariectomised animals treated with progesterone alone (Fig. 1B) or in combination with oestrogen (Fig. 1D and F) when compared with animals treated with oestrogen alone (Fig. 1A). There is an increase in mesometrial compared with antimesometrial AQP1 staining, in the inner circular layer of myometrium, in animals treated with progesterone, in combination with $8 \mathrm{~h}$ of oestrogen (Fig. 1C, antimesometrial; 1D, mesometrial) and $24 \mathrm{~h}$ of oestrogen (Fig. 1E, antimesometrial; 1F, mesometrial). There is an increase in AQP1 immunofluorescence in the mesometrial inner circular layer of ovariectomised animals treated with progesterone and $8 \mathrm{~h}$ of oestrogen (Fig. 1D) compared with other treatment groups.

There is no AQP4 staining in the uterus from any treatment group. Oestrogen (Fig. 2C) and progesterone in combination with oestrogen (Fig. 2D) results are shown.

AQP5 immunofluorescent staining is seen in the luminal epithelial cells from all treatment groups examined, except for the oestrogen treated group (Fig. 3B). In control animals (Fig. 3A), there is apical AQP5 staining. In ovariectomised animals treated with either progesterone alone or in combination with oestrogen there is apical AQP5 staining with no difference between mesometrial (Fig. 3D, progesterone; $3 \mathrm{~F}$, progesterone in combination with oestrogen) and antimesometrial (Fig. 3C, progesterone; 3E, progesterone in combination with oestrogen) luminal epithelial cells.

Controls were carried out with all experimental sections and no staining was observed. Negative controls of myometrium (Fig. 2E) and luminal epithelium (Fig. 2F) from ovariectomised animals treated with progesterone in combination with oestrogen are shown.

\section{Electron microscopy}

Immunogold localisation of AQP1 molecules in myometrium from ovariectomised animal treated with progesterone in combination with oestrogen revealed cytoplasmic localisation in longitudinal muscle cells (Fig. 4A). In 

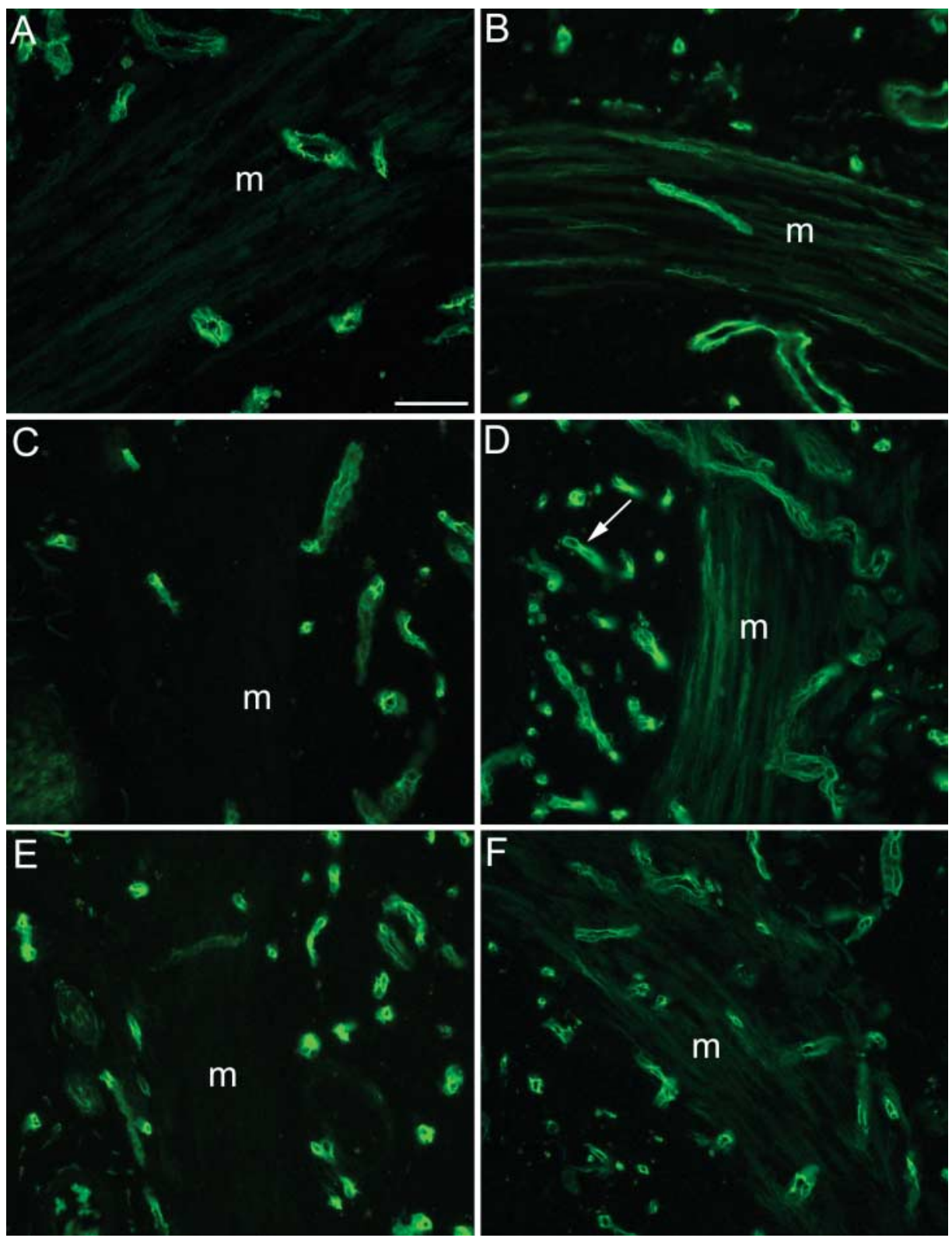

Figure 1 Immunofluorescent images of the rat myometrium showing AQP1 staining. AQP1 is present in the inner circular layer of myometrium ( $\mathrm{m}$ ) in ovariectomised animals treated with oestogen (A), progesterone alone (B) or in combination with oestrogen (C-F). Immunofluorescent staining is increased in the inner circular myometrium at the mesometrial pole in all animals ( $\mathrm{A}$, oestrogen; $\mathrm{B}$, progesterone; $\mathrm{D}$, progesterone and $8 \mathrm{~h}$ oestrogen treatment; $F$, progesterone and $24 \mathrm{~h}$ oestrogen treatment). The antimesometrial myometrium shows very little immunofluorescent staining in progesterone primed animals treated with either 8 (C) or $24 \mathrm{~h}$ (E) of oestrogen. There is an increase in immunofluorescent staining of the inner circular layer of muscle at the mesometrial pole in progesterone primed animals with $8 \mathrm{~h}$ of oestrogen when compared with other treatment groups. Staining of endometrial blood vessels is seen in all treatment groups (arrow). Scale bar, $50 \mu \mathrm{m}$.

circular muscle cells, AQP1 was localised to the plasma membrane (Fig. 4B and C).

AQP5 immunogold labelling was seen in all treatment groups. In ovariectomised control animals (Fig. 5A) and those treated with oestrogen (Fig. 5B), there was mainly cytoplasmic immunogold localisation, with very little staining of the apical plasma membrane. However, in ovariectomised animals treated with progesterone alone (Fig. 5C) or in combination with oestrogen (Figures 5D and E) there was an increase in immunogold particles associated with the apical plasma membrane. There was no difference in immunogold labelling between luminal epithelial cells from mesometrial and antimesometrial poles of the uterus (not shown).

All controls carried out showed no staining. Progesterone in combination with oestrogen is shown as an example (Fig. 4D, smooth muscle cells from inner circular layer of myometrium; Fig. 5F, luminal epithelium). 


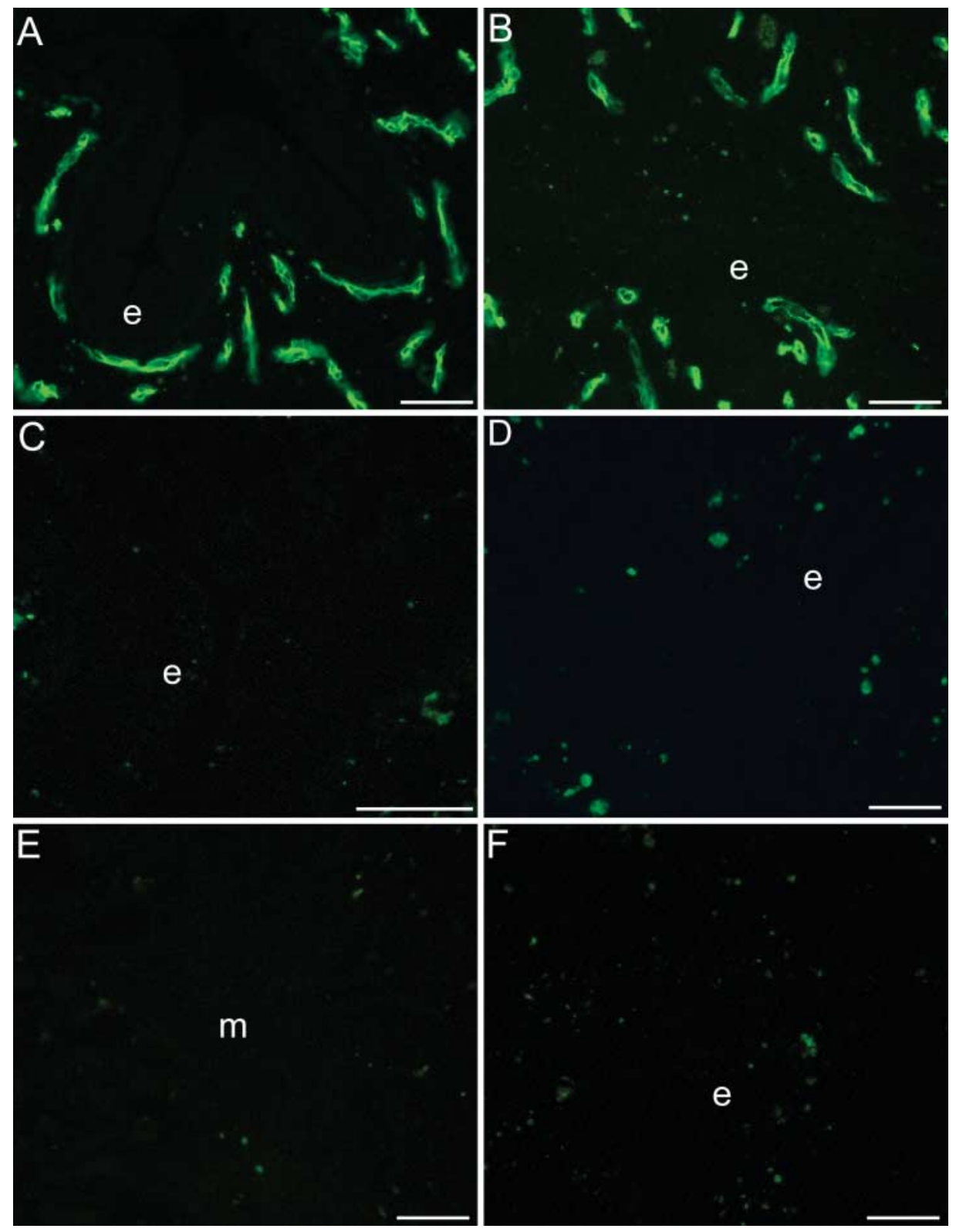

Figure 2 Light micrographs of the uterus from ovariectomised animals treated with oestrogen (A\&C), or progesterone in combination with oestrogen (B, D, E and F). AQP1 immunofluorescent staining is seen in endometrial blood vessels (A and B) but is absent from the uterine luminal epithelium (e). There is no immunofluorescent staining of the uterine stroma or luminal epithelium (e) with anti-AQP4 antibodies (C and D). Negative control images of myometrium (m) and luminal epithelium (e) showing no immunofluorescent staining. Scale bar, $50 \mu \mathrm{m}$.

\section{Discussion}

This study shows for the first time that the increase in apical AQP5 protein expression in rat luminal epithelial cells is controlled by progesterone. It also demonstrates that AQP1 protein levels increased in the inner circular layer of myometrium in the ovariectomised rat treated with progesterone and $8 \mathrm{~h}$ oestrogen.

The increase in AQP5 expression in the apical plasma membrane of luminal epithelial cells of the ovariectomised rat treated with progesterone alone or in combination with oestrogen is consistent with mRNA results in ovariectomised mice treated with progesterone in combination with oestrogen (Richard et al. 2003). However in the rat it appears that progesterone is the minimum requirement to cause such a change, and not progesterone followed by a nidatory oestrogen surge, as is the case in mice (Richard et al. 2003). During normal pregnancy in the rat, a similar increase was seen at the time of implantation however, ovariectomy and hormone administration did not lead to the differential concentration of AQP5 staining seen at the time of implantation. 

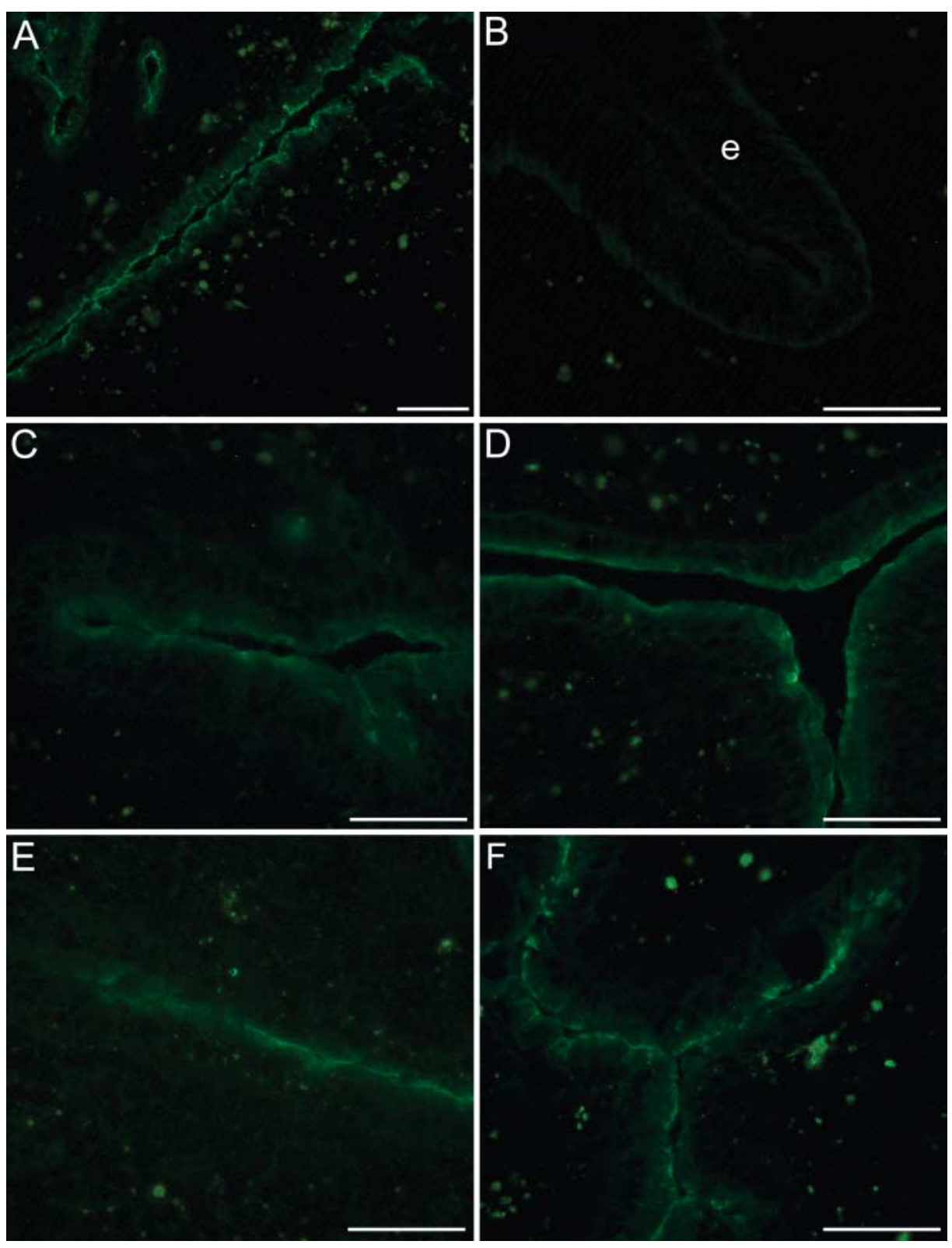

Figure 3 Light micrographs demonstrating AQP5 staining of the rat uterus from control ovariectomised animals treated with oil alone (A), and those treated with oestrogen (B), progesterone alone (C and D) or in combination with oestrogen (E and F). Apical AQP5 immunofluorescent staining is seen in the uterine epithelium from control animals (A). Very little immunofluorescence is seen in the luminal epithelium (e) from the uterus treated with oestrogen (B). AQP5 immunofluorescent staining is seen in the apical part of luminal epithelial cells from ovariectomised animals treated with progesterone alone (C and $\mathrm{D})$ or in combination with oestrogen (E and F). There is no difference in apical AQP5 staining of luminal epithelial cells from mesometrial and antimesometrial poles of the uterus in animals treated with progesterone alone (C, antimesometrial; $\mathrm{D}$, mesometrial) or in combination with oestrogen ( $\mathrm{E}$, antimesometrial; $\mathrm{F}$, mesometrial). Scale bar, $50 \mu \mathrm{m}$.

The fact that the differential concentration of AQP5 staining between mesometrial and antimesometrial poles of the uterine lumen does not exist in ovariectomised animals under any of the hormonal regimes studies, as it does during normal pregnancy (Lindsay \& Murphy $2004 b)$, suggests that this is due to some effect of the blastocyst, perhaps paracrine signals from the blastocyst to the uterine epithelium (Roberts et al. 1989).
The presence of AQP5 proteins in the apical plasma membrane of luminal epithelial cells in response to progesterone, as first demonstrated in light and electron microscopy experiments in the current study, provides a possible mechanism for the reabsorption of luminal fluid seen in the progesterone treated uterus. Since fluid can travel via paracellular or transcellular pathways, and the tight junctions in the progesterone treated uterus are relatively 

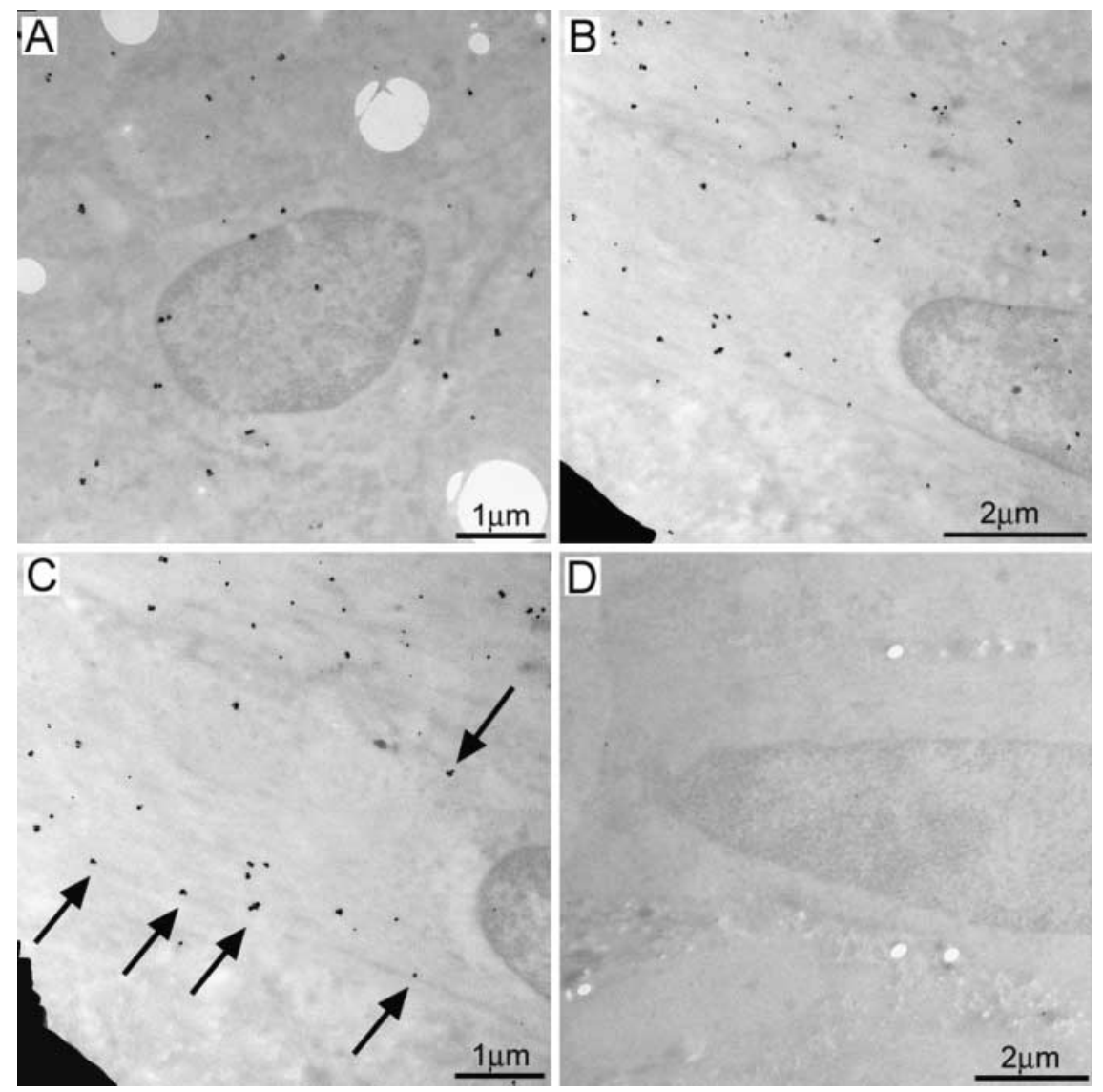

Figure 4 Electron micrographs showing the distribution of AQP1 staining in outer longitudinal (A) and inner circular muscle cells $(B-C)$. There is diffuse gold staining of the outer longitudinal muscle cells (A), while plasma membrane staining (arrows) is seen in muscle cells from the inner circular layer of myometrium (B-C). Negative control (D) showing the absence of gold particles in muscle cells from the inner circular layer.

'tight' (Murphy et al. 1981), fluid may be transported by transcellular means through AQP channels, particularly apical AQP5 channels as are up-regulated in the progesterone treated uterus.

The movement of fluid across an epithelial barrier through AQP channels is a passive process, following osmotic gradients set up by various other solute transporters. In this context, it has been demonstrated that the cystic fibrosis transmembrane conductance regulator (CFTR) and epithelial sodium channel (ENaC) play an important role in the movement of chloride and sodium ions across the endometrial epithelium (Chan et al. 2002). CFTR in the luminal epithelium leads to the secretion of chloride ions into the uterine lumen and the movement of fluid into the lumen during proestrus and early oestrus (Chan et al. 2002). Oestrogen leads to upregulation of CFTR in the uterine epithelium (Rochwerger \& Buchwald 1993, Rowlands et al. 2001), and progesterone, alone or in combination with oestrogen, leads to a down-regulation of CFTR expression (Mularoni et al. 1995). Hence during oestrogen stimulation when chloride ions are secreted into the uterine lumen and there are leaky tight junctions (Murphy et al. 1981), fluid most likely travels into the uterine lumen via paracellular mechanisms. However, during progesterone stimulation where there is a decrease in chloride ion secretion (Mularoni et al. 1995) and an increase in sodium ion secretion into the lumen (Nordenvall et al. 1989), there is an osmotic gradient favoring the movement of water from the lumen to the endometrial stroma. This osmotic gradient, in addition to 'tight' tight junctions seen at this time (Murphy et al. 1981), suggests that fluid movement is via the transcellular pathway involving apical AQP5 channels.

The increase in AQP1 water channels in blood vessels of the endometrial stroma in response to various hormonal regimes is a common feature seen in several organs (Nielsen et al. 1993, Borgnia et al. 1999, King et al. 2000) including the uterus (Lindsay \& Murphy 2004b). Oestrogen causes increases in vascular permeability and stromal oedema (Ljungkvist 1971a, Anderson et al. 1972) and thus the presence of AQP1 molecules in the endometrial stromal blood vessels provides a mechanism for the formation of this stromal oedema.

The presence of AQP1 in the myometrium has previously been demonstrated in the pregnant rat uterus, where there was an increase in the inner circular layer of myometrium and a further increase in this layer of myometrium from the mesometrial compared with the antimesometrial pole (Lindsay \& Murphy 2004a). A similar result was seen in the present work with an increase in AQP1 staining in the inner circular layer of myometrium in response to progesterone alone or in combination with oestrogen. 

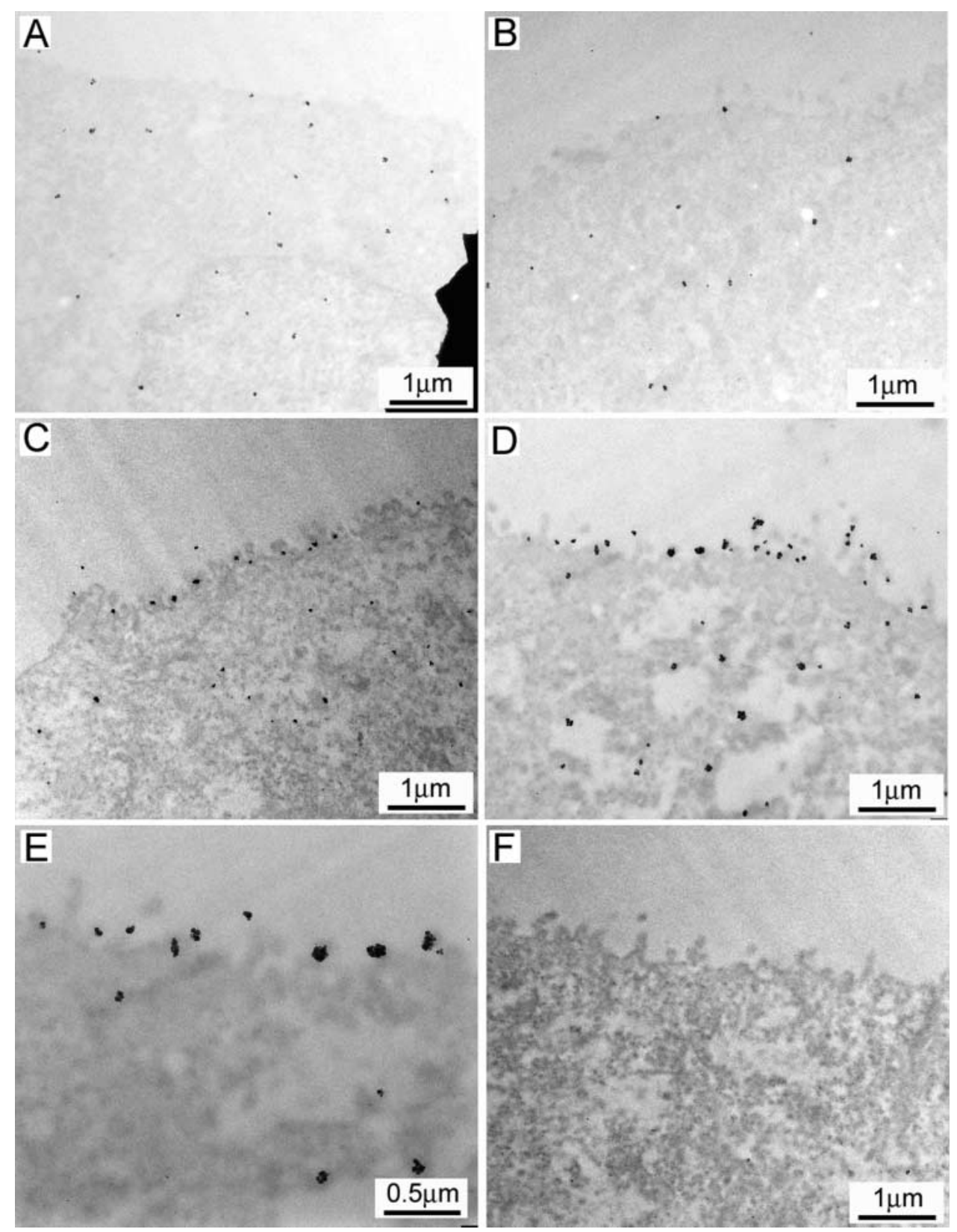

Figure 5 Electron micrographs showing the distribution of AQP5 in the apical portion of luminal epithelial cells from control animals (A), and ovariectomised animals treated with oestrogen (B), progesterone $(C)$ or progesterone in combination with oestrogen (D and $E)$. In control and oestrogen treated animals, there are immunogold particles in the uterine epithelial cell cytoplasm, with very little plasma membrane staining. An increase in immunogold particles associated with the apical plasma membrane is seen in animals treated with progesterone alone or in combination with oestrogen. A higher magnification image (E) shows the immunogold particles associated with the apical plasma membrane. Negative control electron micrograph of luminal epithelial cell from an ovariectomised animal treated with progesterone in combination with oestrogen showing no immunogold staining $(F)$.

Results from this study show that maximal AQP1 staining occurred after $8 \mathrm{~h}$ of oestrogen stimulation to progesterone primed rats, when compared with other hormonal regimes. Similarly, in mice AQP1 protein expression in the myometrium increased in response to oestrogen, and decreased in progesterone treated animals (Richard et al. 2003). The difference in response of rat myometrial AQP1 localisation after 8 and $24 \mathrm{~h}$ of oestrogen treatment to a progesterone-primed rat is consistent with the biphasic response to oestrogen seen in the ovariectomised and progesterone-primed mouse uterus, where maximal AQP1 mRNA expression was found one hour after oestrogen administration (Richard et al. 2003). The time difference between these two studies most likely consists of slight differences in timing between mouse and rat implantation, and the delay in translocation of mRNA into protein. 
Hence, the maximal expression of AQP1 observed in the inner circular layer of myometrium $8 \mathrm{~h}$ after oestrogen administration may represent another aspect of the biphasic response of oestrogen in the progesterone-primed rat.

AQP1 in the inner circular layer of myometrium, seen in the present study, may allow the movement of water from the myometrium into the endometrial stroma, particularly after $8 \mathrm{~h}$ of oestrogen treatment. The decrease in myometrial AQP1 immunofluorescence seen after $24 \mathrm{~h}$ of oestrogen treatment provides further evidence that stromal oedema, which is part of the early response to oestrogen (Anderson et al. 1972), is dependent on AQP1 in the myometrium.

We also observed a differential concentration of AQP1 staining between the mesometrial and antimesometrial pole of the uterus. This suggests that the influence of the asymmetrical AQP1 myometrial expression on blastocyst positioning, as described during early pregnancy (Lindsay \& Murphy 2004b), is not dependent on signaling or the presence of the blastocyst, but is rather an intrinsic effect of the hormonal milieu. It also seems that this could lead to swelling of the uterine myometrium and closing of the lumen, which would explain the appearance of the closed lumen seen in the progesterone treated uterus (Ljungkvist 1971b, Png \& Murphy 2000).

In summary, this is the first study to show that ovarian hormones change the distribution of AQP molecules. Thus, the hormones which regulate implantation are involved in the redistribution of AQP5 to the apical plasma membrane of the luminal epithelial cells and an increase in AQP1 staining of the myometrium. These are the conditions of uterine receptivity, and the present study adds to our understanding of uterine fluid transport and the hormonal mechanisms regulating the switch from paracellular transport mechanisms, controlled by the tight junction complex (Murphy 2000) to transcellular transport by regulation of $\mathrm{AQP}$ expression. It also adds to the events which constitute the plasma membrane transformation (Murphy 2000).

\section{References}

Agre P, Saboori AM, Asimos A \& Smith BL 1987 Purification and partial characterization of the $\mathrm{Mr} 30,000$ integral membrane protein associated with the erythrocyte Rh(D) antigen. Journal of Biological Chemistry 262 17497-17503.

Agre P, Bonhivers M \& Borgnia MJ 1998 The aquaporins, blueprints for cellular plumbing systems. Journal of Biological Chemistry 273 14659-14662.

Agre P, King L, Yasui M, Guggino W, Ottersen O, Fujiyoshi Y, Engel A \& Nielsen S 2002 Aquaporin water channels - from atomic structure to clinical medicine. Journal of Physiology 542 3-16.

Anderson JN, Clark JH \& Peck EJ Jr 1972 The relationship between nuclear receptor-estrogen binding and uterotrophic responses. Biochemical and Biophysical Research Communications $\mathbf{4 8}$ 1460-1468.

Borgnia M, Nielsen S, Engel A \& Agre P 1999 Cellular and molecular biology of the aquaporin water channels. Annual Review of Biochemistry 68 425-458.
Chan LN, Tsang LL, Rowlands DK, Rochelle LG, Boucher RC, Liu CQ \& Chan HC 2002 Distribution and regulation of ENaC subunit and CFTR mRNA expression in murine female reproductive tract. Journal of Membrane Biology 185 165-176.

Claude P \& Goodenough DA 1973 Fracture faces of zonulae occludentes from 'tight' and 'leaky' epithelia. Journal of Cell Biology $\mathbf{5 8}$ 390-400.

Denker BM, Smith BL, Kuhajda FP \& Agre P 1988 Identification, purification, and partial characterization of a novel $\mathrm{Mr} 28,000$ integral membrane protein from erythrocytes and renal tubules. Journal of Biological Chemistry 263 15634-15642.

Enders A \& Schlafke S 1967 A morphological analysis of the early implantation stages in the rat. American Journal of Anatomy $\mathbf{1 2 0}$ $185-226$.

Jablonski EM, McConnell NA, Hughes FM Jr \& Huet-Hudson YM 2003 Estrogen regulation of aquaporins in the mouse uterus: potential roles in uterine water movement. Biology of Reproduction 69 1481-1487.

Julian J, Enders AC, Fazleabas AT \& Carson DD 2005 Compartmental distinctions in uterine Muc-1 expression during early pregnancy in cynomolgous macaque (Macaca fascicularis) and baboon (Papio anubis). Human Reproduction 20 1493-1503.

King LS, Yasui M \& Agre P 2000 Aquaporins in health and disease. Molecular Medicine Today 6 60-65.

Li XJ, Yu HM \& Koide SS 1997 Regulation of water channel gene (AQP-CHIP) expression by estradiol and anordiol in rat uterus. Yao Hsueh Hsueh Pao - Acta Pharmaceutica Sinica 32 586-592.

Lindsay LA \& Murphy CR 2004a Aquaporin-1 increases in the rat myometrium during early pregnancy. Journal of Molecular Histology $3575-79$.

Lindsay LA \& Murphy CR 2004b Redistribution of aquaporins in uterine epithelial cells at the time of implantation in the rat. Acta Histochemica 106 299-307.

Ljungkvist I 1971a Attachment reaction of rat uterine luminal epithelium. III. The effect of estradiol, estrone and estriol on the morphology of the luminal epithelium of the spayed, virgin rat. Acta Societatis Medicorum Upsaliensis 76 139-157.

Ljungkvist I 1971b Attachment reaction of rat uterine luminal epithelium. II. The effect of progesterone on the morphology of the uterine glands and the luminal epithelium of the spayed, virgin rat. Acta Societatis Medicorum Upsaliensis 76 110-126.

Mularoni A, Beck L, Sadir R, Adessi GL \& Nicollier M 1995 Downregulation by progesterone of CFTR expression in endometrial epithelial cells: a study by competitive RT-PCR. Biochemical and Biophysical Research Communications 217 1105-1111.

Murphy CR 2000 Junctional barrier complexes undergo major alterations during the plasma membrane transformation of uterine epithelial cells. Human Reproduction 15 182-188.

Murphy CR, Swift JG, Mukherjee TM \& Rogers AW 1981 Effects of ovarian hormones on cell membranes in the rat uterus. II. Freezefracture studies on tight junctions of the lateral plasma membrane of the luminal epithelium. Cell Biophysics 3 57-69.

Nielsen S, Smith BL, Christensen El \& Agre P 1993 Distribution of the aquaporin CHIP in secretory and resorptive epithelia and capillary endothelia. PNAS $907275-7279$.

Nielsen S, King LS, Christensen BM \& Agre P 1997a Aquaporins in complex tissues. II. Subcellular distribution in respiratory and glandular tissues of rat. American Journal of Physiology 273 C1549-C1561.

Nielsen S, Nagelhus EA, Amiry-Moghaddam M, Bourque C, Agre P \& Ottersen OP $1997 b$ Specialized membrane domains for water transport in glial cells: high-resolution immunogold cytochemistry of aquaporin-4 in rat brain. Journal of Neuroscience $\mathbf{1 7}$ $171-180$.

Nordenvall M, Ulmsten U \& Ungerstedt U 1989 Influence of progesterone on the sodium and potassium concentrations of rat uterine fluid investigated by microdialysis. Gynecologic and Obstetric Investigation 28 73-77. 
Png FY \& Murphy CR 2000 Closure of the uterine lumen and the plasma membrane transformation do not require blastocyst implantation. European Journal of Morphology. 38 122-127.

Richard C, Gao J, Brown N \& Reese J 2003 Aquaporin water channel genes are differentially expressed and regulated by ovarian steroids during the peri-implantation period in the mouse. Endocrinology 144 1533-1541.

Roberts RM, Farin CE \& Imakawa K 1989 Embryonic mediators of maternal recognition of pregnancy. In Blastocyst Implantation, pp 25-30. Ed. K Yoshinaga. Adams Publishing Group Ltd.

Rochwerger L \& Buchwald M 1993 Stimulation of the cystic fibrosis transmembrane regulator expression by estrogen in vivo. Endocrinology 133 921-930.

Rowlands DK, Tsang LL, Cui YG, Chung YW, Chan LN, Liu CQ, James T \& Chan HC 2001 Upregulation of cystic fibrosis transmembrane conductance regulator expression by oestrogen and Bak Foong Pill in mouse uteri. Cell Biology International 25 $1033-1035$.
Song Y, Jayaraman S, Yang B, Matthay MA \& Verkman AS 2001 Role of aquaporin water channels in airway fluid transport, humidification, and surface liquid hydration. Journal of General Physiology $117573-582$.

van Hoek AN, Ma T, Yang B, Verkman AS \& Brown D 2000 Aquaporin-4 is expressed in basolateral membranes of proximal tubule S3 segments in mouse kidney. American Journal of Physiology Renal Fluid and Electrolyte Physiology 278 F310-F316.

Yamamoto T \& Sasaki S 1998 Aquaporins in the kidney: emerging new aspects. Kidney International 54 1041-1051.

Received 4 August 2005

First decision 25 August 2005

Revised manuscript received 11 October 2005

Accepted 20 October 2005 